\title{
Biomarker analysis of the MITO2 phase III trial of first-line treatment in ovarian cancer: predictive value of DNA-PK and phosphorylated ACC
}

Francesco Perrone ${ }^{1, *}$ Gustavo Baldassarre ${ }^{2, *}$ Stefano Indraccolo ${ }^{3, *}$ Simona Signoriello ${ }^{4}$, Gennaro Chiappetta ${ }^{1}$, Franca Esposito ${ }^{5}$, Gabriella Ferrandina $^{6}$, Renato Franco ${ }^{1,15}$, Delia Mezzanzanica7, Maura Sonego ${ }^{2}$, Elisabetta Zulato ${ }^{3}$, Gian F. Zannoni', Vincenzo Canzonieri ${ }^{2}$, Giovanni Scambia ${ }^{6}$, Roberto Sorio², Antonella Savarese $^{8}$, Enrico Breda ${ }^{9}$, Paolo Scollo ${ }^{10}$, Antonella Ferro ${ }^{11}$, Stefano Tamberi ${ }^{12}$, Antonio Febbraro ${ }^{13}$, Donato Natale ${ }^{14}$, Massimo Di Maio ${ }^{1,16}$, Daniela Califano ${ }^{1}$, Giosuè Scognamiglio ${ }^{1}$, Domenica Lorusso', Silvana Canevari', Simona Losito ${ }^{1}$, Ciro Gallo ${ }^{4, * *}$ and Sandro Pignata ${ }^{1, * *}$

${ }^{1}$ Istituto Nazionale per lo Studio e la Cura dei Tumori - Fondazione G.Pascale, IRCCS, Napoli, Italy

${ }^{2}$ Centro di Riferimento Oncologico, IRCCS, Aviano (PN), Italy

${ }^{3}$ Istituto Oncologico Veneto, IRCCS, Padova, Italy

${ }^{4}$ Dipartimento di Salute Mentale, Fisica e Medicina Preventiva, Statistica Medica, Seconda Università, Napoli, Italy

${ }^{5}$ Università di Napoli Federico II, Napoli, Italy

${ }^{6}$ Catholic University, Roma, Italy

${ }^{7}$ Fondazione IRCCS Istituto Nazionale dei Tumori, Milano, Italy

${ }^{8}$ Istituto Nazionale Tumori Regina Elena, IRCCS, Roma, Italy

${ }^{9}$ Ospedale S. Giovanni Calibita Fatebenefratelli, Roma, Italy

${ }^{10}$ Ospedale Cannizzaro, Catania, Italy

${ }^{11}$ Ospedale S. Chiara, Trento, Italy

12 Ospedale Civile, Faenza, Italy

13 Ospedale Fatebenefratelli, Benevento, Italy

14 Ospedale S. Massimo, Penne (PE), Italy

15 Dipartimento di Salute mentale, Fisica e Medicina Preventiva, Anatomia Patologica, Seconda Università, Napoli Italy

${ }^{16}$ Università degli Studi, Torino, Italy

* co-first author

** co-last author

Correspondence to: Sandro Pignata, email: s.pignata@istitutotumori.na.it Keywords: ovarian cancer, phase 3 clinical trial, predictive factors, pACC, DNA-PK

Received: June 11, $2016 \quad$ Accepted: August 03, 2016

Published: September 15, 2016

\section{ABSTRACT}

Background: No biomarker is available to predict prognosis of patients with advanced ovarian cancer (AOC) and guide the choice of chemotherapy. We performed a prospective-retrospective biomarker study within the MITO2 trial on the treatment of AOC.

Patients and methods: MITO2 is a randomised multicentre phase 3 trial conducted with 820 AOC patients assigned carboplatin/paclitaxel (carboplatin: AUC5, paclitaxel: $175 \mathrm{mg} / \mathrm{m}^{2}$, every 3 weeks for 6 cycles) or carboplatin/PLD-pegylated liposomal doxorubicin (carboplatin: AUC5, PLD: $30 \mathrm{mg} / \mathrm{m}^{2}$, every 3 weeks for 6 cycles) as first line treatment. Sixteen biomarkers (pathways of adhesion/invasion, apoptosis, transcription regulation, metabolism, and DNA repair) were studied in 229 patients, in 


\begin{abstract}
a tissue microarray. Progression-free and overall survival were analysed with multivariable Cox model.

Results: After 72 months median follow-up, 594 progressions and 426 deaths were reported; there was no significant difference between the two arms in the whole trial. No biomarker had significant prognostic value. Statistically significant interactions with treatment were found for DNA-dependent protein kinase (DNA-PK) and phosphorylated acetyl-coenzymeA carboxylase (PACC), both predicting worse outcome for patients receiving carboplatin/paclitaxel.

Conclusion: These data show that in presence of DNA-PK or pACC overexpression, carboplatin/paclitaxel might be less effective than carboplatin/PLD as first line treatment of ovarian cancer patients. Further validation of these findings is warranted.
\end{abstract}

\section{INTRODUCTION}

Ovarian cancer includes a heterogeneous group of neoplasms, commonly classified according to clinical, morphological and molecular features. [1] Nevertheless, medical treatment of all types of epithelial ovarian cancer includes carboplatin/paclitaxel as standard backbone, also in combination with bevacizumab. [2-4] Therefore, biomarkers able to identify patients who may benefit from standard or alternative chemotherapy might be useful in clinical practice.

The possibility of grouping ovarian cancer based on molecular portrait has been proposed, $[5,6]$ and biochemical and molecular markers have been studied as prognostic or predictive factors. [7-9] However, only few immunohistochemistry (IHC) studies have been conducted in large series, through consortia or as meta-analysis. [8, $10,11]$ Yet, with the exception of BRCA1/2 mutations to guide administration of PARP inhibitors, $[12,13]$ no biomarker is used in clinical practice.

In 2011, with a 40 months median follow-up, the MITO2 trial showed that carboplatin/PLD was not superior to carboplatin/paclitaxel as first line treatment of ovarian cancer patients. [14] Here, with long-term follow up data, we tested the prognostic and/or predictive role of some biomarkers according to a prospective-retrospective design. [15]

\section{RESULTS}

\section{Long-term efficacy analysis in the whole study population}

After 72 months median follow-up (interquartile range: $60-85)$, in the whole population of 820 patients, there were 594 events for PFS and 426 events for OS analysis. PFS HR for the carboplatin/PLD vs carboplatin/ paclitaxel arm was 0.99 (95\%CI 0.84-1.17; $p=0.93)$; median PFS was 18 (95\%CI 16-23) and 17 months (95\%CI 15-19) in the two arms, respectively. OS HR for the carboplatin/PLD vs carboplatin/paclitaxel arm was
0.94 (95\%CI $0.77-1.13 ; p=0.49)$; median OS was 61 (95\%CI 51-77) and 53 months $(95 \%$ CI $42-70)$ in the two arms, respectively (Figure S1 online).

\section{Biomarker analyses}

Seventeen centres, enrolling 549 patients $(67 \%$ of the whole study), supplied paraffin blocks from the primary tumor of 258 patients; 28 were excluded because tumor had been collected during interval debulking surgery after initiation of chemotherapy or date of collection was unknown. Therefore, 230 were eligible of whom 229 ( $42 \%$ of patients enrolled in the centres participating to the biomarker study and $28 \%$ of the whole study) had at least one biomarker tested and represented the biomarker population (Figure S2 online).

The biomarker population was similar to the overall study population, with a slightly lower rate of patients who were not operated at baseline and who had stage IV disease at diagnosis (Table S2 online). Consistently, both PFS and OS were slightly better in the biomarker population compared to the overall MITO2 population and to all patients enrolled in the centres participating to the biomarker study (Figure S3 online).

Distribution of biomarkers by treatment arm is reported in Table S3 online; due to core losses from the slides during IHC procedure, the number of cases tested ranged from 153 (pAMPK) to 223 (CDK6). No imbalances were observed between treatment arms at the planned significance level of 0.01 .

Moderate pairwise associations (Table S4 online) were only found between pACC and either DNA-PK (Cramer's V $=0.487, p<0.001$ ) or pAMPK (Cramer's V $=0.433, p<0.001)$.

There were no statistically significant associations at the level of 0.001 between patients' or tumors' characteristics and biomarkers' categories (Tables S5-S23 online) except for DNA-PK and residual disease (Table S23 online). Univariate PFS and OS Kaplan-Meier curves for each biomarker are depicted in Figures S4-S41 online.

No biomarker had statistically significant (predefined significance level of 0.01 ) prognostic value for PFS and OS at multivariable analyses (Table 1). 

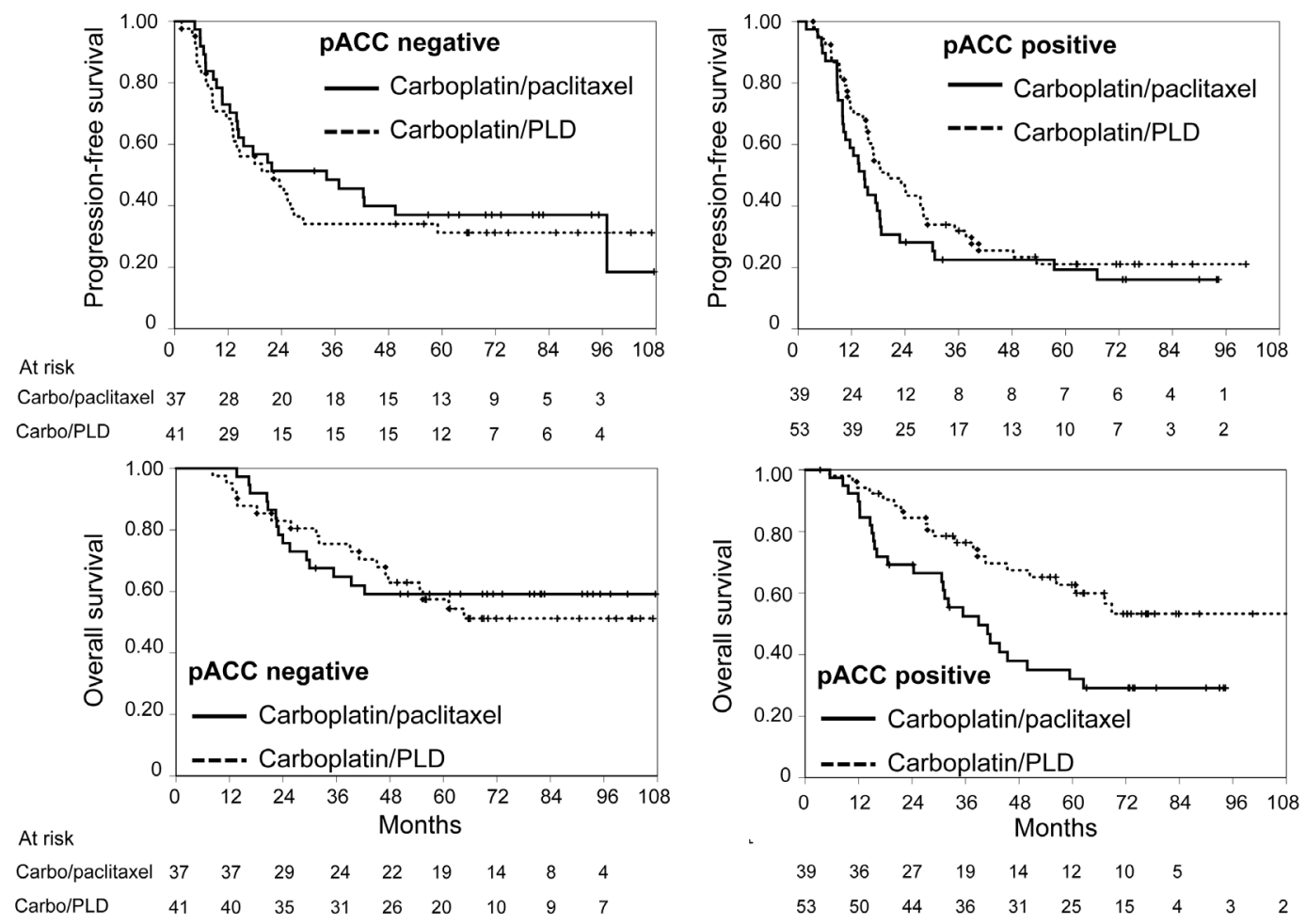

Figure 1: Kaplan-Meier estimated curves of progression-free survival (top) and overall survival (bottom) according to pACC status (negative: graphs on the left, positive: graphs on the right). Solid line: carboplatin/paclitaxel; dashed line: carboplatin/PLD. Vertical lines represent censoring.
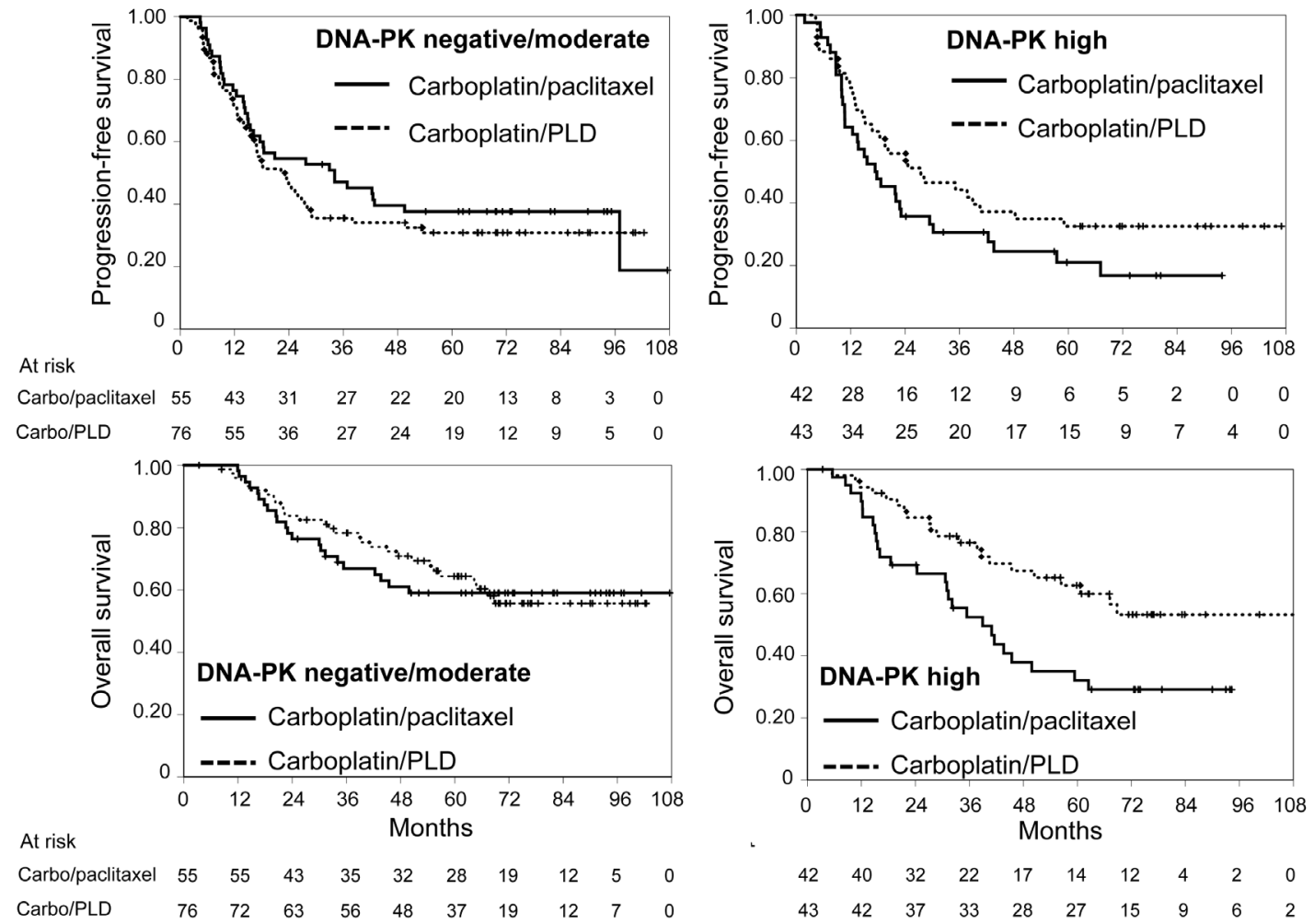

Figure 2: Kaplan-Meier estimated curves of progression-free survival (top) and overall survival (bottom) according to DNA-PK status (negative/moderate: graphs on the left, high: graphs on the right). Solid line: carboplatin/paclitaxel; dashed line: carboplatin/PLD. Vertical lines represent censoring. 
Table 1: Summary of multivariable analyses with candidate prognostic biomarkers

\begin{tabular}{|c|c|c|c|c|c|}
\hline \multirow{2}{*}{$\begin{array}{l}\text { Pathway } \\
\text { Biomarker }\end{array}$} & & \multicolumn{2}{|c|}{ Progression-free survival } & \multicolumn{2}{|c|}{ Overall survival } \\
\hline & & HR $(95 \% \mathrm{Cl})$ & $P$ value & HR (95\% Cl) & $P$ value \\
\hline \multicolumn{6}{|c|}{ Adhesion and invasion } \\
\hline ALCAM & membrane vs cytoplasm & $1.16(0.82-1.66)$ & 0.40 & $0.84(0.52-1.33)$ & 0.46 \\
\hline MCAM & positive vs negative & $0.93(0.65-1.31)$ & 0.66 & $0.79(0.50-1.24)$ & 0.31 \\
\hline \multicolumn{6}{|l|}{ CAV1 } \\
\hline Tumor & positive vs negative & $0.90(0.62-1.32)$ & 0.60 & $0.76(0.45-1.28)$ & 0.30 \\
\hline Stroma & positive vs negative & $1.01(0.75-1.35)$ & 0.97 & $0.82(0.56-1.19)$ & 0.29 \\
\hline Claudin3 & high vs low & $1.07(0.77-1.50)$ & 0.67 & $1.12(0.73-1.70)$ & 0.61 \\
\hline \multicolumn{6}{|l|}{ Apoptosis } \\
\hline cFLIP & positive vs negative & $0.97(0.68-1.40)$ & 0.87 & $1.00(0.64-1.58)$ & 0.99 \\
\hline TRAP & positive vs negative & $0.85(0.60-1.20)$ & 0.35 & $0.77(0.49-1.19)$ & 0.24 \\
\hline \multirow[t]{3}{*}{ BAG3 } & & & 0.28 & & 0.14 \\
\hline & 2 vs $0-1$ & $1.54(0.91-2.62)$ & & $1.94(0.997-3.76)$ & \\
\hline & 3 vs $0-1$ & $1.23(0.80-1.89)$ & & $1.22(0.69-2.13)$ & \\
\hline \multicolumn{6}{|c|}{ Transcription regulation } \\
\hline \multicolumn{6}{|l|}{ HOX B13 } \\
\hline Cytoplasmic & positive vs negative & $1.21(0.86-1.69)$ & 0.27 & $1.07(0.70-1.64)$ & 0.76 \\
\hline Nuclear & positive vs negative & $1.09(0.80-1.51)$ & 0.58 & $1.34(0.89-2.02)$ & 0.16 \\
\hline HMGA2 & $2 / 3$ vs $0 / 1$ & $1.24(0.85-1.81)$ & 0.26 & $1.32(0.82-2.10)$ & 0.25 \\
\hline \multicolumn{6}{|l|}{ CDK6 } \\
\hline \multirow[t]{3}{*}{ Intensity } & & & 0.82 & & 0.11 \\
\hline & high vs low/moderate & $0.88(0.57-1.35)$ & & $0.60(0.35-1.03)$ & \\
\hline & very high vs low/moderate & $0.97(0.62-1.50)$ & & $0.94(0.56-1.58)$ & \\
\hline \multicolumn{2}{|c|}{ Cellular localization } & & 0.21 & & 0.20 \\
\hline & cytoplasm/membrane vs cytoplasm & $1.40(0.94-2.10)$ & & $1.60(0.98-2.63)$ & \\
\hline & cytoplasm/nucles vs cytoplasm & $0.86(0.44-1.65)$ & & $1.15(0.52-2.56)$ & \\
\hline \multicolumn{6}{|l|}{ Metabolism } \\
\hline \multirow[t]{3}{*}{ Leptin receptor } & & & 0.31 & & 0.048 \\
\hline & $10 \% / 50 \%$ vs $<10 \%$ & $1.35(0.83-2.21)$ & & $1.98(1.10-3.56)$ & \\
\hline & $>50 \%$ vs $<10 \%$ & $0.92(0.64-1.31)$ & & $0.95(0.59-1.53)$ & \\
\hline
\end{tabular}

At multivariable analyses of putative predictive biomarkers (Table 2), the predictive value role was confirmed for pACC (for both PFS and OS) and for DNA-PK (only for PFS). In both cases the presence of the biomarker appears to reduce the effect of the carboplatin/ paclitaxel combination (Figures 1 and 2).

\section{DISCUSSION}

We tried to find out molecular tumor characteristics that may ultimately be useful for prognostic prevision and to guide the choice of chemotherapy, within the MITO2 randomized clinical trial of first-line chemotherapy for patients with AOC. None of 16 putative biomarkers had significant independent prognostic value; the expression of pACC and DNA-PK had a statistically significant interaction with treatment identifying patients who had less benefit from the carboplatin/paclitaxel combination as compared to the carboplatin/PLD one.

A biomarker analysis performed within a prospective clinical trial, with a common protocol for archival tumor samples management and statistical analysis independent of the laboratories (reducing risk of ex-post hypotheses adjustment), has a level of evidence immediately behind that of trials prospectively planned for biomarker analysis. [15] Nevertheless, our study has a priori limitations. First, previous evidence had several weaknesses, deriving from small, retrospective, selected, non-protocol-driven data sets. Second, tumor collection was not mandatory in MITO2, and an attrition bias was expected, that further increased due to random missing data produced by technical limitations of TMA technology; therefore, our study is powered for medium-large prognostic effects. Third, a conservative statistical approach was required to reduce the risk of false positive results coming from multiple testing.

Two biomarkers, DNA-PK and pACC, had a statistically significant interaction with treatment, their overexpression being predictive of worse efficacy of the carboplatin/paclitaxel treatment. This is consistent with the hypothesis that high DNA-PK expression should identify patients more resistant to paclitaxel therapy. [16] DNA$\mathrm{PK}$ and $\mathrm{pACC}$ expression have different functions, since DNA-PK is the catalytic subunit of a complex necessary for the Non-Homologous End Joining (NHEJ) DNA repair activity, [17] while ACC is the enzyme that converts acetyl-CoA in malonyl-CoA; the latter is phosphorylated and inhibited by AMP-activated protein kinase (AMPK), 

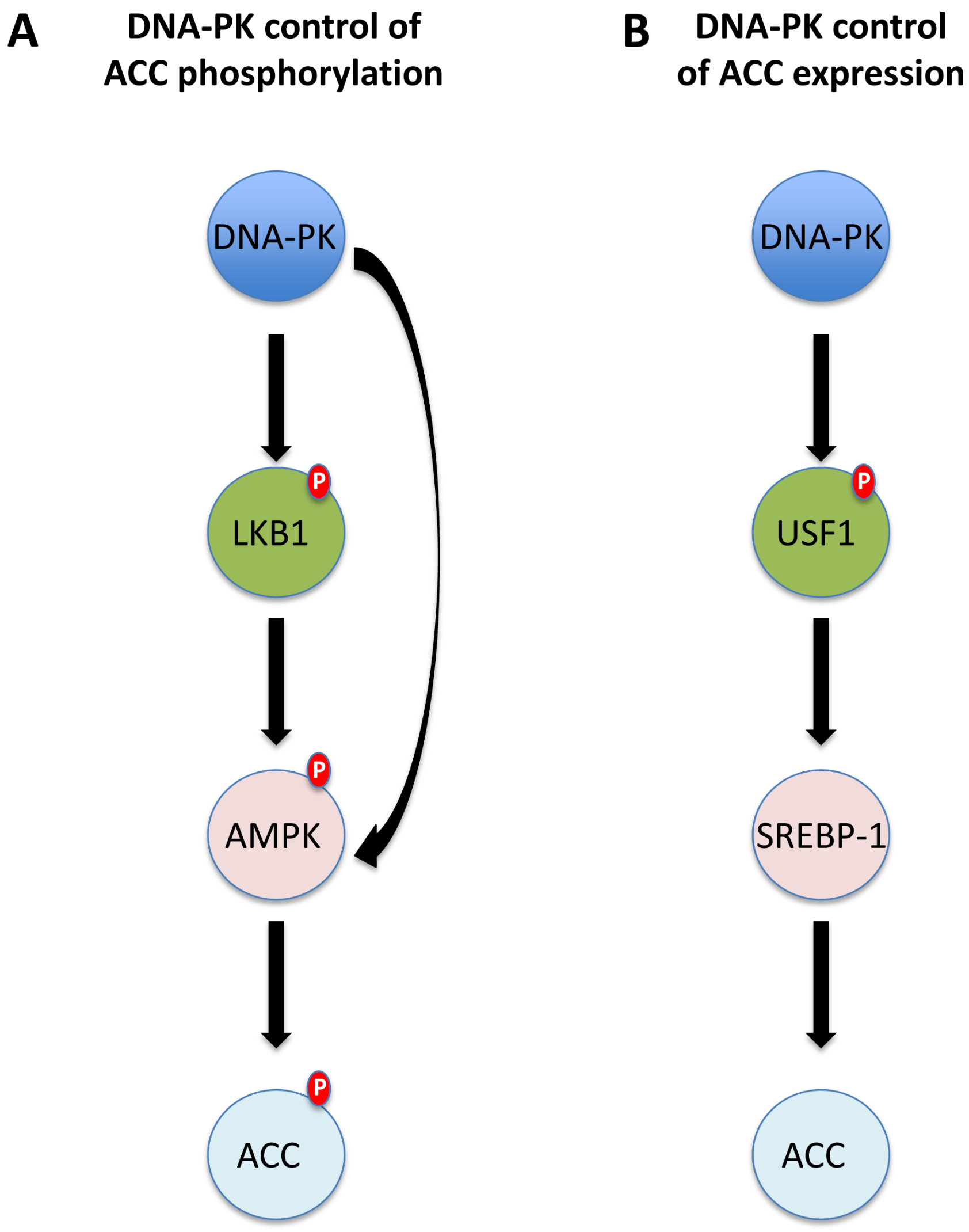

Figure 3: Possible molecular links between DNA-PKs and pACC expression. A. DNA-PK could phosphorylate and activate AMPK directly, or indirectly via LKB1. AMPK activation eventually results in high ACC phosphorylation. B. DNA-PK phosphorylates the transcription factor USF1. This event is necessary for the proper expression of FAS (and ACC) by SREBP-1. Higher levels of pACC are in this case the consequence of higher levels of the total protein. 
Table 2: Summary of multivariable analyses with candidate prognostic and predictive biomarkers

\begin{tabular}{|c|c|c|c|c|c|c|c|}
\hline \multirow{2}{*}{$\begin{array}{l}\text { Pathway } \\
\text { Biomarker }\end{array}$} & & \multicolumn{3}{|c|}{ Progression-free survival } & \multicolumn{3}{|c|}{ Overall survival } \\
\hline & & HR $(95 \% \mathrm{CI})$ & $\begin{array}{c}P \\
\text { value }\end{array}$ & $\begin{array}{c}\text { Interaction } \\
P \text { value }\end{array}$ & $\mathrm{HR}(95 \% \mathrm{CI})$ & $\begin{array}{c}P \\
\text { value }\end{array}$ & $\begin{array}{c}\text { Interaction } \\
P \text { value }\end{array}$ \\
\hline \multicolumn{8}{|l|}{ Apoptosis } \\
\hline P53 & high vs low & $0.71(0.51-0.99)$ & 0.045 & 0.74 & $1.00(0.64-1.58)$ & 0.99 & 0.59 \\
\hline \multicolumn{8}{|l|}{ Metabolism } \\
\hline pAMPK & positive vs negative & $1.00(0.67-1.50)$ & 0.99 & 0.73 & $1.13(0.67-1.89)$ & 0.65 & 0.75 \\
\hline \multirow[t]{3}{*}{$\mathrm{pACC}$} & positive vs negative & $1.15(0.79-1.68)$ & 0.47 & 0.03 & $1.21(0.77-1.91)$ & 0.41 & 0.005 \\
\hline & arm: carboplatin/paclitaxel & $1.79(1.00-3.20)$ & & & $2.34(1.20-4.58)$ & & \\
\hline & arm: carboplatin/PLD & $0.77(0.45-1.31)$ & & & $0.69(0.36-1.33)$ & & \\
\hline \multicolumn{8}{|l|}{ DNA repair } \\
\hline Stathmin & high vs neg/moderate intensity & $0.88(0.62-1.25)$ & 0.47 & 0.68 & $0.88(0.55-1.41)$ & 0.59 & 0.25 \\
\hline \multirow[t]{3}{*}{ DNA-PK } & high vs neg/moderate intensity & $0.74(0.52-1.06$ & 0.10 & 0.048 & $0.88(0.57-1.35)$ & 0.55 & 0.09 \\
\hline & arm: carboplatin/paclitaxel & $1.12(0.65-1.95)$ & & & & & \\
\hline & arm: carboplatin/PLD & $0.51(0.30-0.86)$ & & & & & \\
\hline
\end{tabular}

a sensor of cellular energy charge and a metabolic master switch. [18] In the present study, DNA-PK and pACC expression were correlated (Cramer's V $=0.487, p<$ 0.001 ) and there are at least two likely explanations for this correlation (Figure 3). First, DNA-PK could activate both LKB1 and AMPK, resulting in high ACC phosphorylation. $[17,19]$ Second, DNA-PK is a master regulator of fatty acids synthesis and its expression and activity is necessary for the proper regulation of the transcription factors USF 1 and SERBP1 that control the expression of ACC and FAS (Fatty Acid Synthase). [20] In any case, it is now clear that DNA-PK is necessary for several biological functions other than the control of NHEJ DNA-repair and, among these, regulation of cellular metabolism via transcription regulation seems to be of primary relevance. $[17,21]$ AMPK also plays an important role in this setting; its activation, indeed, is necessary for the proper induction of doxorubicin-mediated death in several models, possibly through the control of autophagy; [22, 23] on the contrary, the same mechanism has been shown to protect cells from paclitaxel induced cell death, enhancing glycolysis during mitosis and preventing mitotic cell death. [24] These data strongly suggest that higher DNA-PK expression can prompt higher AMPK activity, causing higher pACC levels, and ultimately identifies ovarian cancer cells resistant to paclitaxel and sensitive to doxorubicin treatment.

Alternatively, it should be considered that paclitaxel action on interphase microtubules alters proteins transport and re-localization and may prevent nuclear localization of the enzymes involved in DNA-repair activity including DNA-PK, potentiating the activity of cytotoxic drug like platinum. [25-27] It is therefore possible that cells expressing high levels of DNA-PK are less sensitive to paclitaxel-induced DNA-PK cytoplasmic retention and more resistant to the effect of the carboplatin/paclitaxel combination.

In conclusion, we believe that our findings unveil a new pathway in ovarian cancer that might play an important role in the response of therapy. Additional work is needed to substantiate this hypothesis and to definitely clarify the involved mechanisms; also, further validation in larger series or prospective trials is warranted. DNAPK and pACC might be first-in-kind biomarkers for personalizing the choice of chemotherapy regimen in ovarian cancer.

\section{MATERIALS AND METHODS}

MITO2 is registered at clinicaltrials.gov NCT00326456. Trial details have been previously reported. [14] In the present long-term efficacy analysis, PFS and OS curves were reported according to the KaplanMeier method and were compared with the Log-rank test. Median estimates and HR, with $95 \%$ confidence intervals (CI) were also reported. All the analyses were performed according to intention-to-treat.

Following the approval of study amendments by Ethical Committee and patients' consent, tissue microarrays (TMA), prepared at the Pathology Unit of the NCI Naples (see page 5 online), were sent to seven laboratories involved in the study to test 16 putative biomarkers, some of which had more than one measurement, leading to 19 analyses. Biomarkers were classified according to their prevalent biological activity in 5 different groups: adhesion/invasion, apoptosis, transcription regulation, metabolism, DNA repair. Expected/hypothesized role and evidence or preliminary data for each studied biomarker are detailed online (pages 5-10 online). Methods for biomarker testing procedures are detailed in Table S1 online. Investigators involved in TMA preparation and analysis were blinded to the assigned treatment and patients' outcome.

The biomarker population was defined as the subgroup of patients for whom at least one biomarker result was available. Different two-tailed significance levels were applied to different biomarker analyses to 
partially adjust for multiple comparisons. Three planned conventional levels of $\mathrm{P}$ value, more or less conservative, were used according to the specific aim and the number of planned tests $(0.001,0.01,0.05)$.

Baseline characteristics, and PFS and OS outcomes of the biomarker population were described, without statistical testing, to allow an informal comparison with the whole study population and the population of patients enrolled by the centres that participated in tumor block collection.

Association of biomarkers with baseline characteristics of the patients and the tumors were described and tested with the Chi-square test or the Wilcoxon rank sum test or the Kruskal-Wallis test, as appropriate, using a significance level of 0.001. PFS and OS curves were drawn with the Kaplan-Meier method.

Biomarkers were categorized in two or more levels according to predefined cut-offs. Pairwise associations between biomarkers were assessed by means of Cramer's $\mathrm{V}$ ranging from 0 (no association ) to 1 (two variables fully concordant, give the same information). Statistical significance of pairwise associations was assessed with Chi-square test, using a significance level of 0.001 .

The analysis of the prognostic role of each biomarker was adjusted by predefined clinical prognostic factors. Endpoints were PFS and OS. Each biomarker was added to a multivariable Cox's model with treatment arm, stage, residual disease, grading and age as covariates and prognostic effect was tested at a significance level of 0.01 .

For P53, pAMPK, pACC, Stathmin, and DNAPK previous data suggested a modifying effect on the treatment with taxane/anthracycline (see appendix online). Therefore, a predictive role had to be assessed and it was planned a priori to test the biomarker*treatment interaction, using the conventional significance level of 0.05 . If the interaction test was not statistically significant the prognostic effect was evaluated as above.

\section{CONFLICTS OF INTEREST}

The Authors have declared no conflict of interest.

\section{FUNDING}

This work was partially supported by Integrated Therapeutics Group Schering-Plough Italy; AIRC (Italian Association for Cancer Research) grant IG5776; Ministero della Salute (RF-2010-2309704). The supporters did not play any role in protocol and manuscript writing, trial conduction, data analysis and interpretation. The study was sponsored by National Cancer Institute of Naples.

\section{REFERENCES}

1. Kurman RJ and Shih Ie M. Molecular pathogenesis and extraovarian origin of epithelial ovarian cancer-shifting the paradigm. Human pathology. 2011; 42:918-931.

2. Burger RA, Brady MF, Bookman MA, Fleming GF, Monk BJ, Huang H, Mannel RS, Homesley HD, Fowler J, Greer BE, Boente M, Birrer MJ, Liang SX and Gynecologic Oncology G. Incorporation of bevacizumab in the primary treatment of ovarian cancer. The New England journal of medicine. 2011; 365:2473-2483.

3. Perren TJ, Swart AM, Pfisterer J, Ledermann JA, PujadeLauraine E, Kristensen G, Carey MS, Beale P, Cervantes A, Kurzeder C, du Bois A, Sehouli J, Kimmig R, Stahle A, Collinson F, Essapen S, et al. A phase 3 trial of bevacizumab in ovarian cancer. The New England journal of medicine. 2011; 365:2484-2496.

4. Thigpen T, duBois A, McAlpine J, DiSaia P, Fujiwara K, Hoskins W, Kristensen G, Mannel R, Markman M, Pfisterer J, Quinn M, Reed N, Swart AM, Berek J, Colombo N, Freyer G, et al. First-line therapy in ovarian cancer trials. International journal of gynecological cancer. 2011;21:756762.

5. Bookman MA, Gilks CB, Kohn EC, Kaplan KO, Huntsman D, Aghajanian C, Birrer MJ, Ledermann JA, Oza AM and Swenerton KD. Better therapeutic trials in ovarian cancer. Journal of the National Cancer Institute. 2014; 106:dju029.

6. Kohn EC, Romano S and Lee JM. Clinical implications of using molecular diagnostics for ovarian cancers. Annals of oncology. 2013; 24 Suppl 10:x22-26.

7. de Graeff P, Crijns AP, de Jong S, Boezen M, Post WJ, de Vries EG, van der Zee AG and de Bock GH. Modest effect of p53, EGFR and HER-2/neu on prognosis in epithelial ovarian cancer: a meta-analysis. British journal of cancer. 2009; 101:149-159.

8. Wang Y, Wang D and Ren M. Prognostic value of HER-2/ neu expression in epithelial ovarian cancer: a meta-analysis. Tumour biology. 2014; 35:33-38.

9. Xu L, Cai J, Yang Q, Ding H, Wu L, Li T and Wang Z. Prognostic significance of several biomarkers in epithelial ovarian cancer: a meta-analysis of published studies. Journal of cancer research and clinical oncology. 2013; 139:1257-1277.

10. Kobel M, Madore J, Ramus SJ, Clarke BA, Pharoah PD, Deen S, Bowtell DD, Odunsi K, Menon U, Morrison C, Lele S, Bshara W, Sucheston L, Beckmann MW, Hein A, Thiel FC, et al. Evidence for a time-dependent association between FOLR1 expression and survival from ovarian carcinoma: implications for clinical testing. An Ovarian Tumour Tissue Analysis consortium study. British journal of cancer. 2014; 111:2297-2307.

11. Sieh W, Kobel M, Longacre TA, Bowtell DD, deFazio A, Goodman MT, Hogdall E, Deen S, Wentzensen N, Moysich KB, Brenton JD, Clarke BA, Menon U, Gilks CB, Kim A, Madore J, et al. Hormone-receptor expression and ovarian cancer survival: an Ovarian Tumor Tissue Analysis consortium study. The Lancet Oncology. 2013; 14:853-862.

12. Kaye SB, Lubinski J, Matulonis U, Ang JE, Gourley C, 
Karlan BY, Amnon A, Bell-McGuinn KM, Chen LM, Friedlander M, Safra T, Vergote I, Wickens M, Lowe ES, Carmichael J and Kaufman B. Phase II, open-label, randomized, multicenter study comparing the efficacy and safety of olaparib, a poly (ADP-ribose) polymerase inhibitor, and pegylated liposomal doxorubicin in patients with BRCA1 or BRCA2 mutations and recurrent ovarian cancer. Journal of clinical oncology. 2012; 30:372-379.

13. Ledermann J, Harter P, Gourley C, Friedlander M, Vergote I, Rustin G, Scott CL, Meier W, Shapira-Frommer R, Safra T, Matei D, Fielding A, Spencer S, Dougherty B, Orr M, Hodgson D, et al. Olaparib maintenance therapy in patients with platinum-sensitive relapsed serous ovarian cancer: a preplanned retrospective analysis of outcomes by BRCA status in a randomised phase 2 trial. The Lancet Oncology. 2014; 15:852-861.

14. Pignata S, Scambia G, Ferrandina G, Savarese A, Sorio R, Breda E, Gebbia V, Musso P, Frigerio L, Del Medico P, Lombardi AV, Febbraro A, Scollo P, Ferro A, Tamberi $\mathrm{S}$, Brandes A, et al. Carboplatin plus paclitaxel versus carboplatin plus pegylated liposomal doxorubicin as firstline treatment for patients with ovarian cancer: the MITO2 randomized phase III trial. Journal of clinical oncology. 2011; 29:3628-3635.

15. Simon RM, Paik $S$ and Hayes DF. Use of archived specimens in evaluation of prognostic and predictive biomarkers. J Natl Cancer Inst. 2009; 101:1446-1452.

16. Sonego M, Schiappacassi M, Lovisa S, Dall'Acqua A, Bagnoli M, Lovat F, Libra M, D'Andrea S, Canzonieri V, Militello L, Napoli M, Giorda G, Pivetta B, Mezzanzanica D, Barbareschi M, Valeri B, et al. Stathmin regulates mutant p53 stability and transcriptional activity in ovarian cancer. EMBO molecular medicine. 2013; 5:707-722.

17. Goodwin JF and Knudsen KE. Beyond DNA repair: DNAPK function in cancer. Cancer Discov. 2014; 4:1126-1139.

18. Hardie DG, Ross FA and Hawley SA. AMPK: a nutrient and energy sensor that maintains energy homeostasis. Nature reviews Molecular cell biology. 2012; 13:251-262.

19. Sanli T, Steinberg GR, Singh G and Tsakiridis T. AMPactivated protein kinase (AMPK) beyond metabolism: a novel genomic stress sensor participating in the DNA damage response pathway. Cancer biology \& therapy. 2014; 15:156-169.
20. Wong RH, Chang I, Hudak CS, Hyun S, Kwan HY and Sul HS. A role of DNA-PK for the metabolic gene regulation in response to insulin. Cell. 2009; 136:1056-1072.

21. Goodwin JF, Kothari V, Drake JM, Zhao S, Dylgjeri E, Dean JL, Schiewer MJ, McNair C, Jones JK, Aytes A, Magee MS, Snook AE, Zhu Z, Den RB, Birbe RC, Gomella LG, et al. DNA-PKcs-Mediated Transcriptional Regulation Drives Prostate Cancer Progression and Metastasis. Cancer Cell. 2015; 28:97-113.

22. Ji C, Yang B, Yang YL, He SH, Miao DS, He L and Bi ZG. Exogenous cell-permeable C6 ceramide sensitizes multiple cancer cell lines to Doxorubicin-induced apoptosis by promoting AMPK activation and mTORC1 inhibition. Oncogene. 2010; 29:6557-6568.

23. Shin DH, Choi YJ and Park JW. SIRT1 and AMPK mediate hypoxia-induced resistance of non-small cell lung cancers to cisplatin and doxorubicin. Cancer research. 2014; 74:298-308.

24. Domenech E, Maestre C, Esteban-Martinez L, Partida D, Pascual R, Fernandez-Miranda G, Seco E, Campos-Olivas R, Perez M, Megias D, Allen K, Lopez M, Saha AK, Velasco G, Rial E, Mendez R, et al. AMPK and PFKFB3 mediate glycolysis and survival in response to mitophagy during mitotic arrest. Nat Cell Biol. 2015; 17:1304-1316.

25. Komlodi-Pasztor E, Sackett D, Wilkerson J and Fojo T. Mitosis is not a key target of microtubule agents in patient tumors. Nat Rev Clin Oncol. 2011; 8:244-250.

26. Mitchison TJ. The proliferation rate paradox in antimitotic chemotherapy. Mol Biol Cell. 2012; 23:1-6.

27. Poruchynsky MS, Komlodi-Pasztor E, Trostel S, Wilkerson J, Regairaz M, Pommier Y, Zhang X, Kumar Maity T, Robey R, Burotto M, Sackett D, Guha U and Fojo AT. Microtubule-targeting agents augment the toxicity of DNAdamaging agents by disrupting intracellular trafficking of DNA repair proteins. Proceedings of the National Academy of Sciences of the United States of America. 2015; 112:1571-1576. 